\title{
Urostomy Obstruction, CTCAE
}

National Cancer Institute

\section{Source}

National Cancer Institute. Urostomy Obstruction, CT CAE. NCI Thesaurus. Code C143911.

A disorder characterized by blockage of the urostomy. 